\title{
La escalera del palacio de los condes de Miran- da en Peñaranda de Duero, Burgos
}

Jorge Martínez Montero

Universidad de León

\begin{abstract}
RESUMEN. Una de las mayores aportaciones a la arquitectura del Renacimiento, viene de la mano de la innovación y difusión de un novedoso elemento constructivo, hasta el momento escasamente analizado: la escalera. Como eje estructural y funcional en el conjunto arquitectónico del edificio, va a contar con la adquisición de nuevos valores técnicos, ornamentales y simbólicos. Buena muestra del reconocimiento y puesta en valor de dicho elemento, es la magnífica escalera del palacio de los Zúñiga-Avellaneda en Peñaranda de Duero, uno de los mejores ejemplos de la arquitectura civil burgalesa del siglo XVI.

Palabras clave: Arquitectura civil, siglo XVI, palacio, escalera renacentista, Burgos.

ABSTRACT. One of the most important contributions to the renaissance architecture comes with the innovation and spreading of a new constructive element, until the present shortly analized: the staircase. As a structural and functional element on the whole architectural of the building, the staircase is going to acquire new technical, ornamental and symbolical values. A good example of the recognition and the valuation of this element, is the magnificent staircase of the Zúñiga-Avellaneda's palace in Peñaranda de Duero, one of the best examples of the 16th century civil architecture of Burgos.

Key Words: Civil architecture, 16th century, palace, renaissance staircase, Burgos.
\end{abstract}

En el extremo sur de la localidad burgalesa de Peñaranda de Duero, junto a la Colegiata y en plena plaza, se levanta una de las mansiones nobiliarias más importantes de la arquitectura renacentista hispana, fiel al reflejo del dominio señorial de los condes de Miranda, uno de los linajes castellanos más señeros del siglo XVI.

\section{LA LABOR DE MECENAZGO DE LOS ZÚÑIGA-AVELLANEDA}

Don Francisco de Zúñiga-Avellaneda y Velasco (†1536), sexto señor de Peñaranda, virrey de Navarra, caballero de la orden del Toisón de Oro y tercer conde de Miran- da del Castañar (Salamanca), es considerado el principal promotor e impulsor de la fábrica, hecho que se desprende de la inscripción del dintel de la portada principal del palacio, en el que reza lo siguiente: "ESTE EDEFICIO MANDO HASER EL ILUSTRE S. DON FRANCISCO DE SUÑIGA DE AVELLANEDA TERCERO CONDE DE MIRANDA S. DE LA CASA DE AVELLANEDA I DE AÇA"1.

${ }^{1}$ Para profundizar en el estudio de la familia Zúñiga-Avellaneda, véase: $P$. DOMINGO JIMENO, «La villa ducal de Peñaranda de Duero y sus relaciones con el Monasterio Franciscano de La Aguilera», Boletín de la 
Hijo de Don Pedro de Zúñiga Avellaneda y de Doña Catalina de Velasco, contrae matrimonio con la hija de los duques de Maqueda, Doña María Enríquez de Cárdenas, promotora de la construcción de grandes obras en la villa, como la prestigiosa Colegiata de Santa Ana o el Hospital de la Piedad. Fruto de su matrimonio, nace el que será el cuarto conde de Miranda, Don Francisco de Zúñiga-Avellaneda de Cárdenas, inminente seguidor del papel fundador de su padre ${ }^{2}$.

Institución Fernán González, CLXVI, 1966, pp. 101-106, artículo de escaso rigor documental en el que se establecen diversas confusiones genealógicas. Mayor veracidad comparten los estudios de I. CADIÑANOS BARDECI, Arquitectura fortificada en la provincia de Burgos, Burgos, 1987, pp. 237-238; «Peñaranda de Duero: Notas de historia y arte», Biblioteca. Estudio e Investigación, VIII, 1993, pp. 114-115; M. C. PORRAS GIL, «Estética y humanismo en la Familia Zúñiga Avellaneda», Renacimiento del Duero, Biblioteca. Estudio e Investigación, XVIII, 2003, pp. 117-141.

El título del condado de Miranda del Castañar, se inauguró en la persona de Don Diego de Zúñiga Guzmán, casado con Doña Aldonza de Avellaneda, quien por su riqueza e influencia política fue nombrado primer conde de Miranda en 1457. Dicho condado, comprendía además de la villa de Miranda del Castañar, los pueblos de Cepeda, Herguijuela de la Sierra, Madroñal, Monforte, Mogarraz, San Martín del Castañar, Casas del Conde, Garcibuey, Santibáñez de la Sierra, Santa María del Llano, Pinedas y Molinillo. Don Diego, cedió el mayorazgo a su hijo, Don Pedro de Zúñiga Avellaneda, segundo conde de Miranda, señor de Aza y Peñaranda (cargos que engloban un total de veinte villas y treinta lugares, por los que percibía alcabalas, diezmos, portazgos y diversos derechos más). Véase: G. RODRÍGUEZ LÓPEZ, «Apuntes biográficos sobre los señores y duques de Béjar», Contribución al estudio de la historia de Béjar. Colección de estudios y documentos, Béjar, 1919, p. 82; J. Álvarez VILLAR, La villa condal de Miranda del Castañar, Salamanca, 1972.

2 I. CADIÑANOS BARDECI, «Peñaranda de Duero: Notas de historia y arte»..., p. 119. Del testamento otorgado por Doña María Enríquez de Cárdenas, a 26 de enero de 1544, se deduce la existencia de al menos otros dos hijos más, Don Pedro Núñez de Avellaneda, abad de San Isidoro de León, y Don Gaspar de Zúñiga, cardenal de Sevilla.

\section{FÁBRICA Y AUTORÍA DEL PALACIO.}

Muchos han sido los autores que se han aventurado a delimitar el período constructivo del palacio. En la década de los veinte, Torres Balbás cree que se erigió en la primera mitad del siglo XVI, mientras que, en los años sesenta, Domingo Jimeno, ante un error genealógico, afirma que se mandó edificar a mediados del citado siglo ${ }^{3}$.

Aunque todo apunta a fechar el inmueble en el primer tercio del siglo XVI, lo cierto es que a pesar de deducir que la labor de fábrica tiene lugar con anterioridad a la muerte del promotor, acaecida el cinco de octubre de 1536, hasta el momento y ante la falta de documentación que lo corrobore, no se ha establecido una fecha exacta del inicio y fin de las obras, desconociendo de igual manera, el proceso constructivo del mismo ${ }^{4}$.

La atribución o autoría de las trazas y posterior ejecución del palacio, constituye aún hoy un episodio sin desvelar. Desde que Lampérez y Romea lanzara la hipótesis de considerar a Francisco de Colonia o a alguno de sus discípulos o imitadores, entre los que aparecería la figura del burgalés Juan de Vallejo, como autores materiales

\footnotetext{
${ }^{3}$ L. TORRES BALBÁs, «De cómo desaparecen los antiguos palacios de la nobleza castellana», Arquitectura, I, 1923, p. 107; P. DOMINGO Jimeno, Peñaranda de Duero, honor de villas castellanas, Burgos, 1961, p. 10.

${ }^{4}$ Una de las últimas aportaciones al dudoso campo cronológico en el que se ve inserto el palacio, viene de la mano del estudio de la heráldica. El historiador Domínguez Casas da como buena una fecha bastante anterior al año 1531 para dar por concluido o inaugurado el Palacio, basándose en la siguiente afirmación: "En ningún lugar del Palacio aparecen las armas de Zúñiga y Avellaneda rodeadas por el collar del Toisón de Oro, Orden caballeresca en la que ingresó el III Conde de Miranda del Castañar en el XX Capítulo, celebrado en la Catedral de Tournai los días 3 a 5 de diciembre de 1531, bajo la presidencia del emperador Carlos $V^{\prime \prime}$ R. DOMínGUEZ CASAS, «Heráldica en el arte del Renacimiento: Burgos y el Sur Provincial», Renacimiento del Duero, Biblioteca. Estudio e Investigación, XVIII, 2003, p. 244.
} 
del conjunto, muchos han sido los que se han sumado al mismo planteamiento, dando como buena tal afirmación ${ }^{5}$.

Sin embargo, en las últimas décadas, maestros de la talla de Nicolás de Vergara, Felipe Bigarny, o el propio Luis de Vega, han aparecido como posibles autores de diversas partes del palacio, de carácter más ornamental, entre las que se encontraría la bella fachada principal o la señorial escalera monumental.

\section{DESCRIPCIÓN ARTÍSTICA}

Fiel ejemplo de un palacio urbano del Renacimiento, impulsado por un promotor de claras convicciones humanísticas, surge como núcleo generador de un espacio urbano en vías de crecimiento.

De planta rectangular, al interior se accede por mediación de una entrada acodada, estancia rectangular que da paso a un gran patio central de sección cuadrangular, en torno al cual se distribuyen las distintas dependencias; muchas de ellas cubiertas por ostentosos artesonados y ornamentadas con frisos de yeserías ${ }^{6}$. Al exterior, la facha-

${ }^{5}$ V. LAMPÉReZ y RomeA, «El Palacio de los condes de Miranda en Peñaranda de Duero (Burgos)» Boletín de la Sociedad Española de Excursiones, 1912, p. 150. Afirmación basada en una semejanza estilística (superposición de cornisa y arco, composición presente en la portada de la Pellejería de la Catedral de Burgos) y mera relación cronológica, ya que Francisco de Colonia estuvo trabajando activamente en toda la comarca desde 1511 hasta 1542.

J. CAMÓN AZNAR, La arquitectura plateresca, I, Madrid, 1945, p. 82; recoge la opinión de Lampérez, aunque cree que probablemente los constructores y decoradores, pertenezcan al ciclo de las primeras obras renacentistas de la Catedral de Sigüenza. F. CHUECA GoITIA, Arquitectura del siglo XVI, Ars Hispaniae, XI, Madrid, 1953, p. 64; afirma que se adscribe a la escuela de Francisco de Colonia, concretamente a los artistas que trabajaron en la Pellejería, en las obras de San Esteban y Santa Dorotea.

${ }^{6}$ Muchos de los artesonados fueron realizados por alarifes mudéjares, residentes todos ellos en la propia villa. F. MARÍAS FRANCO, «Pedro de Gumiel, Francisco da principal se abre a una gran plaza, mientras que la parte trasera se orientaría a un bello jardín, cercado por una muralla de marcadas resonancias militares ${ }^{7}$ (Fig. $n^{\mathbf{0}} 1$ ).

De enorme volumen y magnificencia, su longitudinal fachada pétrea se estructura en dos niveles bien diferenciados, en los que la decoración aparece concentrada en partes muy concretas: portada adintelada, un tanto descentrada respecto al conjunto, y sucesión de ventanas correspondientes a la planta noble ${ }^{8}$.

La portada principal, aparece enmarcada por jambas en jaspe de Espejón, y motivos agrutescados en los extremos; sobre ella, un arco de medio punto ciego en el que aparecen las armas de los promotores. A ambos lados, dos hombres de armas con

de Carabaña, la Universidad de Alcalá y el mito del estilo Cisneros", Boletín del Museo e Instituto "Camón Aznar", LVIII, 1994, p. 70.

Magníficas yeserías de influencias mudéjares decoran algunas de las estancias del palacio, similares a las existentes en la sala capitular de la catedral toledana, y a las que cubren el salón del Paraninfo de la Universidad de Alcalá de Henares, ambas realizadas durante el arzobispado de Jiménez de Cisneros (14951517). M. L. CONCEJO DíEZ, El arte mudéjar en Burgos y su provincia. Tesis Doctoral inédita, Facultad de Geografía e Historia, Universidad Complutense de Madrid, II, 1999, pp. 667-685; F. MARÍAS FRANCO, «El ornato en el ámbito del arzobispado de Toledo», L'Invention de la Renaissance: la réception des formes "Á l'Antique" au début de la Renaissance. Actas de los coloquios de Tours, del 1 al 4 de junio de 1994, París, 2003, p. 199.

${ }^{7}$ M. J. ZAPARAÍN YÁÑEZ, Desarrollo artístico de la comarca arandina siglos XVII y XVIII, II, Burgos, 2002, p. 254. Jardín, calificado de anticuario y extremadamente lujoso, en el que al menos se tiene constancia de la presencia de dos grandes fuentes de mármol y jaspe.

${ }^{8}$ La disposición interna que presentaba originariamente la planta baja del palacio ha sido muy modificada, sin embargo, la planta noble mantiene bastante bien la estructura de muchas de sus salas o salones, de entre los que merece la pena resaltar el afamado Salón de Embajadores. Con su singular celosía calada y afiligranada chimenea, de la que muchos han afirmado tiene, ciertas influencias francesas, relacionadas con el estilo del reinado de Francisco I. 
sus respectivas lanzas flanquean los escudos; mientras que en la parte superior del propio vano se suceden una serie de molduras horizontales, en cuyo centro se sobrepone un nuevo arco en el que se inserta un busto clásico alusivo a la representación mitológica de Hércules, símbolo de la virtud y la defensa de la casa ${ }^{9}$.

Un amplio zaguán da entrada al solemne patio, espacio configurado mediante la superposición de dos niveles de arcadas muy diferenciadas, en las que se mezclan dos tradiciones, comunes en el tiempo una de la otra: el cuerpo inferior denota connotaciones tardogóticas, mientras que el superior es deudor de un lenguaje estrictamente renaciente. Este último, guarda una estrecha vinculación con la galería superior del soriano Monasterio de Santa María de Huerta (1531-1533), el vallisoletano de Don Francisco de los Cobos (1524-1535), o el Palacio de los Dueñas en Medina del Campo (1528-1530), meras analogías o paralelismos entre diversos edificios, con fases constructivas muy parejas en el tiempo ${ }^{10}$ (Fig. $\mathrm{n}^{\mathrm{o}}$ 2).

-

${ }^{9}$ Para conocer de manera detallada la distribución, estructura, decoración y carga simbólica de la fachada, véase: E. CARAZO, «El Palacio de los condes de Miranda en Peñaranda de Duero», Academia, LXXXV, 1997, pp. 524-526; R. J. PAYO HeRnANZ, «Burgos», Casas y Palacios de Castilla y León, Valladolid, 2002, p. 65; M. C. PORRAS GIL, «Estética y humanismo en la Familia Zúñiga Avellaneda»..., pp. 135-136. Sobre la reiterada presencia de Hércules en el mundo del Renacimiento: R. LÓPEZ TORRIJOS, «Representaciones de Hércules en obras religiosas del siglo XVI», Boletín del Seminario de Estudios de Arte y Arqueología, XLVI, 1980, pp. 293-308.

${ }^{10}$ M. D. CAMPOS SÁNCHEZ-BORDONA, «La influencia del humanismo en el claustro del monasterio de Santa María de Huerta (Soria)», Humanismo y Císter. Actas del I Congreso Nacional de Humanistas Españoles, León, 1996, pp. 601-620; F. MARÍAS FRANCO, «La obra renacentista del Claustro de los Caballeros de Santa María la Real de Huerta», Monjes y Monasterios. El Cister en el medievo de Castilla y León, Valladolid, 1998, pp. 289-295.
Imbuido de un profundo italianismo, con una clara diagonalidad en su composición, la interconexión en altura de ambos pisos, tiene lugar mediante una excepcional escalera monumental, elemento de marcado carácter humanístico, indiscutible dentro del nobiliario ceremonial de la época ${ }^{11}$.

\section{IV.LA ESCALERA}

Ejemplo pionero en la evolución de la escalera moderna hispana, paradigma de las escaleras monumentales interiores, constituye una de las primeras fases de experimentación en su gestación y posterior desarrollo ${ }^{12}$.

Su intencionada localización diagonal respecto al zaguán de entrada, en el ángulo noreste del patio, acentúa aún más su marcado sentido de la ocultación, propio

-

${ }^{11}$ M. T. FERNÁNDEZ MADRID, El mecenazgo de los Mendoza en Guadalajara, Guadalajara, 1991, p. 96. Cree ver en el palacio un puro italianismo, en la línea de los edificios erigidos por sus parientes los Mendoza, en Guadalajara. Muy cercano a las construcciones de Lupiana y Sigüenza.

${ }^{12}$ Como único artículo realizado hasta el momento, centrado en la evolución de la escalera renacentista en el ámbito burgalés, se ha de destacar el siguiente: J. MARTÍNEZ MONTERO, «La escalera en la arquitectura civil burgalesa del Renacimiento", Actas del IV Congreso Nacional de Historia de la Construcción, II, Cádiz, 2005, pp. 759-770.

En el panorama europeo, los trabajos centrados en el estudio de la escalera renacentista también escasean: P. FRANKL, (1914), Principios fundamentales de la Historia de la Arquitectura. Barcelona, 1981, pp. 126-133; N. PEVSNER, (1943), Breve historia de la arquitectura europea, Madrid, 1994, pp. 236-238; H. WETHEY, «Escaleras del primer renacimiento español», Archivo Español de Arte, Madrid, CXLVIII, 1964, pp. 295-305; A. CHASTEL, «Un miembro privilegiado de la arquitectura», L'escalier dans l'architecture de la Renaissance. Actas de los coloquios de Tours, del 22 al 26 de mayo de 1979, París, 1985, pp. 7-8; J. GUILLAUME, «Génesis de la escalera moderna», L'escalier dans l'architecture de la Renaissance..., pp. 9-14; A. BUSTAMANTE GARCÍA, «La influencia italiana en la escalera española del Renacimiento», L'escalier dans l'architecture de la Renaissance..., pp. 171174; C. SÁnCHEZ-Robles BeltráN, Composición: La escalera I. La innovación tipológica en arquitectura: las nuevas escaleras post-medievales, Valencia, 1988. 
del carácter intimista de las residencias nobiliarias del Renacimiento ${ }^{13}$ (Fig. $\mathrm{n}^{\mathrm{o}} 3$ ). En detrimento de su estricto valor funcional, acaba adquiriendo nuevos matices ornamentales y simbólicos, formando parte, como elemento de solemnidad y distinción indispensable, del ceremonial aristocrático del momento; práctica protocolaria consistente en la entrada triunfal de los nobles señores y su séquito, en el interior de sus moradas, descabalgando previamente en el zaguán, ascendiendo por la propia escalera, y finalizando su recorrido en las zonas más nobles del palacio.

Vanagloriada y ensalzada por algunos, como el propio Lampérez y Romea, quien afirma de ella: "Escalera magnífica, amplia, monumental, regia. El señorío castellano labró pocas obras que puedan comparársela. Enorme el espacio, anchos los tiros, riquísimas las puertas, ventanas, antepechos y pilarotes, todo de valiente trazado y profusa ornamentación, cuyo esplendor se sobrepasa en la techumbre..." ha llegado a despertar verdadera tristeza en quienes la vieron languidecer, sumida en un impasible estado de abandono ${ }^{14}$ (Fig. $n^{-} 4$ ).

Realizada en piedra de cantería, aparece ubicada en la panda norte del patio principal, permitiendo la comunicación entre la planta baja y el primer piso; tipológicamente es una escalera claustral de tres tramos, distribuidos en una media de dos

\footnotetext{
13 D. Villalobos AlONSO, El debate clasicista y el Palacio de Fabio Nelli, Valladolid, 1992, pp. 17-38. Con respecto a las nociones de la localización de las escaleras en los palacios del Renacimiento, véase los capítulos dedicados a lo que él denomina "espacio diagonal, dual y ocultado".

${ }^{14}$ V. LAMPÉREZ Y ROMEA, «El Palacio de los condes de Miranda en Peñaranda de Duero (Burgos)»..., pp. 147-148; I. GIL, Memorias históricas de Burgos y su provincia, Burgos, 1913, pp. 239-240; A. BYNE \& M. STAPLEY, Spanish architecture of the sixteenth century, Nueva York, 1917, pp. 106-129; L. TORRES BALBÁs, «De cómo desaparecen los antiguos palacios de la nobleza castellana»..., p. 107.
}

rellanos y once peldaños por tiro respectivamente.

Se accede a ella a través de una solemne embocadura a modo de enmarque arquitectónico, constituida por un gran arco carpanel bíforo, fragmentado en su interior en otros dos vanos que aparecen decorados con motivos nervados y que descansan directamente sobre una estilizada columna de jaspe rojo ${ }^{15}$.

La caja de la escalera, de amplias magnitudes, constituye uno de los primeros ejemplos de caja abierta en el Renacimiento burgalés, novedad que hace posible la colocación de un majestuoso artesonado como sistema de cubrición ${ }^{16}$ (Fig. $n^{0}$ 5). Artesonado de madera tallada, sobre un friso de yesería y una cornisa de mocárabes, elementos todos ellos de raigambre mudéjar, fusionados con otros como la presencia en los arrocabes de motivos heráldicos, portados por parejas de estilizados ángeles, o la sucesión de unos dieciséis bustos clásicos, propios del nuevo lenguaje renacentista, al más puro estilo borgoñón, en el que se ve inmerso el palacio; ornamentación en la que queda una vez más patente el poder del linaje Zúñiga-Avellaneda ${ }^{17}$.

Una puerta de marcado lenguaje clásico y un vano adintelado, permite el acceso

\footnotetext{
${ }^{15}$ Este enmarque recuerda al que presidía el acceso a la escalera del desaparecido palacio arzobispal de Alcalá de Henares, obra del genial Alonso de Cobarrubias (1524-1534). Véase: E. TORMO, El Palacio de Alcalá de Henares, Madrid, 1917; M. VEGA Y MARCH, El Archivo de Alcalá de Henares, Biblioteca selecta de Arte Español, V, Madrid, 1923.

${ }^{16}$ Un riguroso y exhaustivo estudio del artesonado de la escalera, y de su expoliación a finales del siglo XIX, es el realizado por E. CARAZO, «El Palacio de los condes de Miranda en Peñaranda de Duero»..., pp. 529-536.

${ }^{17}$ Sobre el análisis de éstos y otros bustos, presentes en la rica decoración del palacio, véase: M. J. REDONDO CANTERA, «Escultura del Renacimiento en las Aguas Durolenses», Renacimiento del Duero, Biblioteca. Estudio e Investigación, XVIII, 2003, pp. 295-297.
} 
al interior del lienzo de muro en talud sobre el que discurren cada una de las rampas, aspecto que permite la entrada y posterior aprovechamiento del espacio generado bajo las mismas.

En el ascenso por el primer tramo, una puerta comunica con una de las estancias intermedias, al finalizar el segundo tiro, una característica ventana de asiento aporta mayor iluminación al espacio, mientras que una ventana geminada sobre columna jaspeada, tondo con busto clásico flanqueado por erotes y decoración acasetonada, acaba por realizar las funciones de tribuna en la planta noble ${ }^{18}$. Elemento característicamente renaciente, que contribuye a ampliar la diafanidad del espacio, aportando un mayor cariz de monumentalidad al conjunto.

La desembocadura está formada por un gran vano adintelado, enmarcado por dos columnas y ornamentado con yeserías distribuidas en una sucesión de molduras con motivos vegetales y geométricos (Fig. $n^{0}$ 6).

La belleza y esplendor con que luce actualmente la escalera, es el resultado de un complicado proceso de restauración, llevada a cabo desde 1950, ya que como anteriormente se ha expuesto, el palacio se vio inmerso en un oscuro período de de-

-

${ }^{18}$ I. Río DE LA HOZ, El escultor Felipe Bigarny, Valladolid, 2001, p. 331. Cree ver una relación entre los balaustres de la ventana superior de la escalera, enlazados por mediación de un motivo sogueado, y los existentes en la escalinata de la cercana iglesia de Santa María del Campo. También hace ver la semejanza que presenta con la iglesia de Santo Tomás de Haro, La Rioja, en el empleo de casetones cuadrados con rosetas clásicas en el esviaje del propio vano geminado. En el campo de la ornamentación, las similitudes con el riojano Palacio de Casalarreina, son claras.

Estas son algunas de las analogías que presentan varias obras, contemporáneas en el tiempo y relacionadas con el taller del propio Felipe Bigarny, en relación con el Palacio de los condes de Miranda. gradación a lo largo de sus dos últimos siglos de historia ${ }^{19}$.

Balaustradas, barandillas y pasamanos, junto a la colocación de motivos heráldicos y remates ornamentales, entre los que se encuentra un característico león erguido, portando el escudo acuartelado de los Zúñiga-Avellaneda, han sido elementos añadidos con posterioridad, acordes al gusto del momento ${ }^{20}$.

De autoría muy discutida, la obra se llevaría a cabo entre los años 1520-1535 por un espléndido maestro de obras, emplazado bajo las órdenes de un adelantado mecenas, sabedor de la estética humanística del Renacimiento y profundo conocedor de la concepción espacial en la arquitectura civil del momento (Fig. no 7).

La trascendencia de dicha escalera, reside en el cúmulo de relaciones e influencias del que es objeto, escalera claustral de tres tramos de caja abierta, como la de la casa del doctor Diego Beltrán, más conocida como palacio de los Dueñas, en Medina del

${ }^{19}$ A. BYNE \& M. STAPLEY, Spanish architecture of the sixteenth century..., p. 118. Anuncia la desaparición de la balaustrada de la escalera (posiblemente fuera de jaspe) a consecuencia de la instalación de una sierra mecánica sobre ella, fruto de la ubicación de un taller de reparación de carretas en el interior del palacio, principal causante del deterioro de la misma.

${ }^{20}$ Escudo compuesto por cuatro carteles: el de los Zúñiga, representado por una banda negra en campo de plata, orlada con ocho eslabones de cadena.

Los Bazán, cuyo cuarto lo ocupan quince jaqueles o juego de ajedrez en oro y negro, teniendo por orla ocho aspas en campo rojo.

Los Avellaneda, en el tercer cuarto, descendientes del linaje López de Haro, cuentan como armas con lobos cebados en corderos sangrientos, sobre campo de oro y orla de ocho aspas.

Los Cárdenas, ocupando el último cuartel, mediante la representación de dos lobos sin cebo, sobre campo rojo, y ocho veneras como orla.

Para profundizar en el estudio de la heráldica presente en el palacio, véase: R. DomíngueZ CASAS, «Heráldica en el arte del Renacimiento: Burgos y el Sur Provincial»..., pp. 240-244. 
Campo; su análoga y contemporánea en el tiempo, proyectada y realizada entre los años 1528 y 1530 por el arquitecto Luis de Vega, junto a los maestros Pedro de la Piedra y Diego de Soba, muestra un gran número de semejanzas estilísticas y formales ${ }^{21}$ (Fig. nº 8 y 9).

Otras, como la del madrileño palacio arzobispal de Alcalá de Henares (15241534) o la del hospital de la Santa Cruz (1521-1522) en Toledo, realizadas ambas en la década de los veinte por Alonso de Covarrubias, también guardan un cúmulo de coincidencias, patentes en elementos materiales, ornamentales y estructurales (Fig. $\mathrm{n}^{\mathrm{o}}$ 10 y 11). Valga como muestra, el empleo intencionado de la imitación de sillares en el muro, variante decorativa característica, aunque no exclusiva, del foco toledano; presente en ejemplos muy tempranos, como la escalera del vallisoletano colegio de San Gregorio (1488-1496), o la escalera capitular de la Catedral de León (1523-1534) ${ }^{22}$.

La colocación de balaustradas caladas, labradas y bien trabajadas, en las que la presencia reiterada de animales protectores, portadores en ocasiones del escudo del linaje, se hace imprescindible (Fig. no 12 ). Buena muestra de ello, se aprecia en ejemplos tan conocidos como el palacio salman-

\section{-}

${ }^{21}$ E. GARCía CHICO, «El Palacio de los Dueñas de Medina del Campo», Boletín del Seminario de Estudios de Arte y Arqueología, XVI, 1950, pp. 87-110; J. URREA FERNÁNDEZ, «El arquitecto Luis de Vega (h. 14951562)», A introduçâo da Arte da Renascença na Península Ibérica, Coimbra, 1981, pp. 162-164.

${ }^{22}$ A cerca de la escalera catedralicia, véase: M. D. CAMPOS SÁNCHEZ-BORDONA, Juan de Badajoz y la arquitectura del Renacimiento en León, León, 1993, pp. 333343 tino de San Boal, el palacio de los marqueses de Ulloa, en la Mota del Marqués, Valladolid, el citado palacio de los Dueñas, o el palacio de los marqueses de Grajal de Campos, en León ${ }^{23}$.

\section{CONCLUSIONES}

La intensa tarea de mecenazgo y patrocinio desempeñada por los condes de Miranda en la villa de Peñaranda de Duero, ha permitido que pueda contar con uno de los mejores ejemplos de arquitectura señorial, equiparable a los más bellos e insignes palacios renacentistas del momento. A través del presente análisis, centrado en un mayor acercamiento al conocimiento de la escalera, como un elemento arquitectónico común en todos ellos, se pretende poner de manifiesto sus múltiples valores, técnicos, estructurales, ornamentales, simbólicos o artísticos.

En último término, y como parte de la meticulosa labor de restauración que se realizó hace ya algunos años en el palacio, me gustaría dejar constancia del buen hacer en la conservación y puesta en valor de la misma, devolviéndole toda su belleza y esplendor, contribuyendo así a ensalzar su papel original, como uno de los elementos indispensables en el conjunto del edificio.

\footnotetext{
${ }^{23}$ Sobre este último palacio, consúltese la monografía: M. D. CAMPOS SÁNCHEZ-BORDONA, Arquitectura y mecenazgo de La Casa de Grajal de Campos, León, 1995, en especial las páginas dedicadas al análisis de la escalera monumental, pp. 88-93.
} 


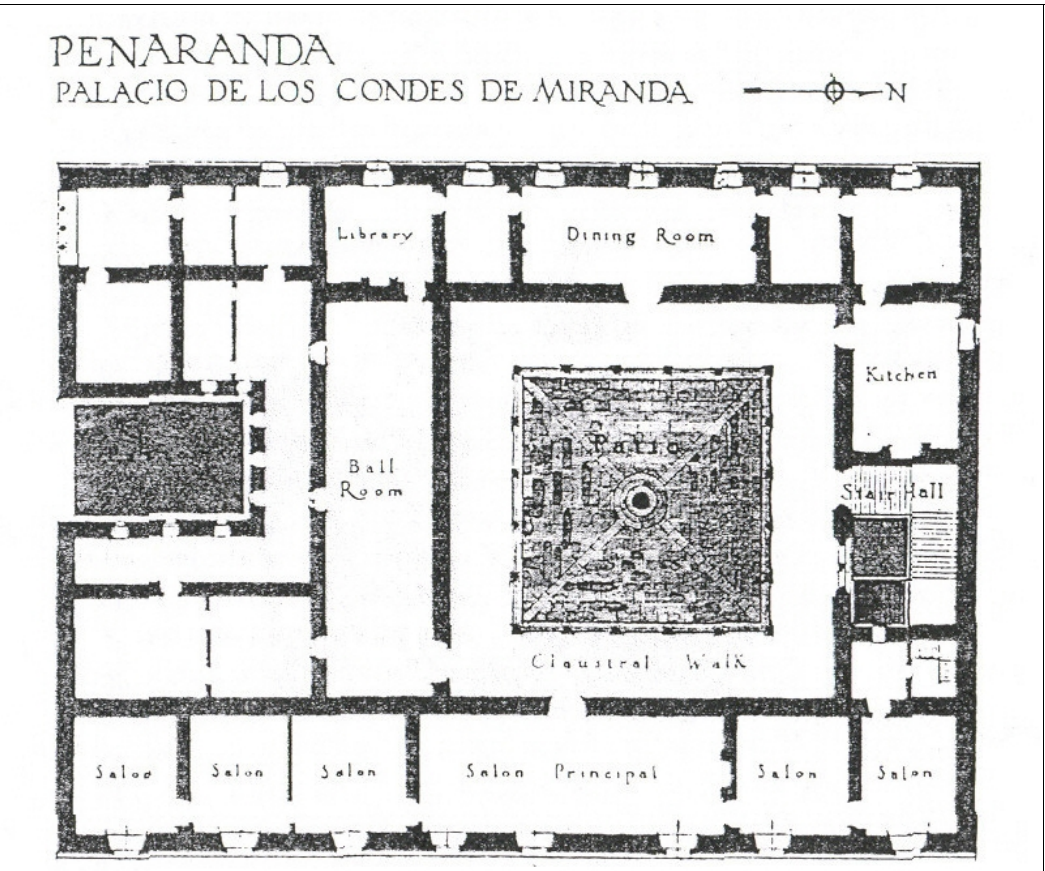

- Fig. 1. Palacio de los condes de Miranda. Planta. A. BynE \& M. STAPLeY, Spanish architecture of the sixteenth century, Nueva York, 1917, p. 110.

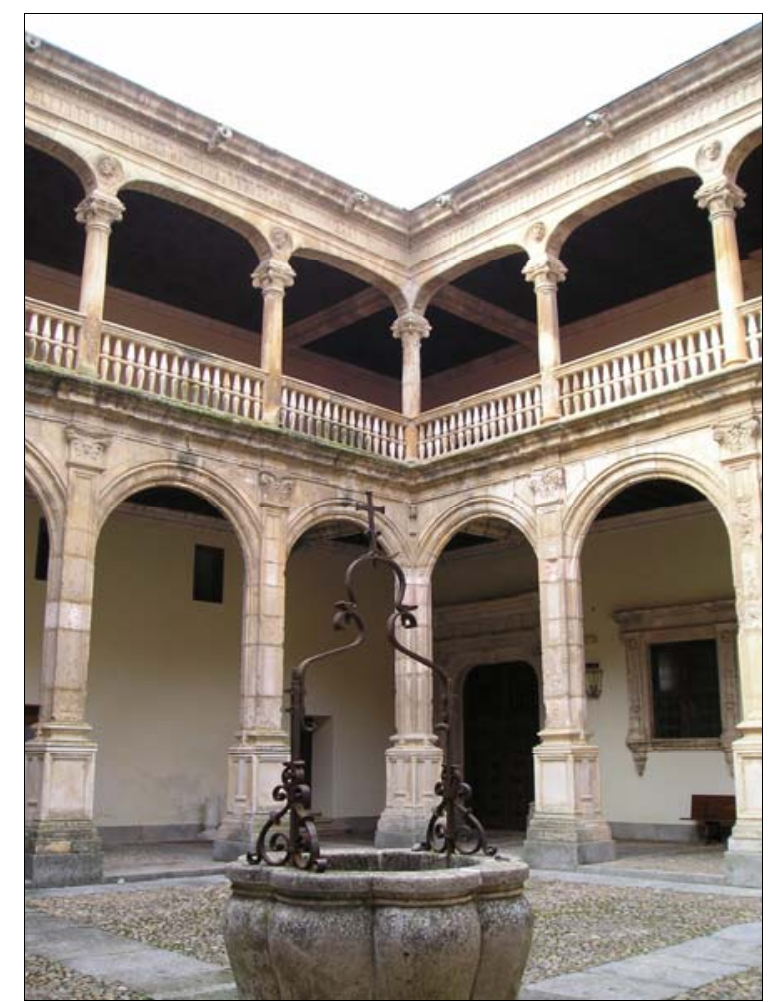

- Fig. 2. Palacio de los condes de Miranda. Vista actual del patio. Fotografía del autor. 


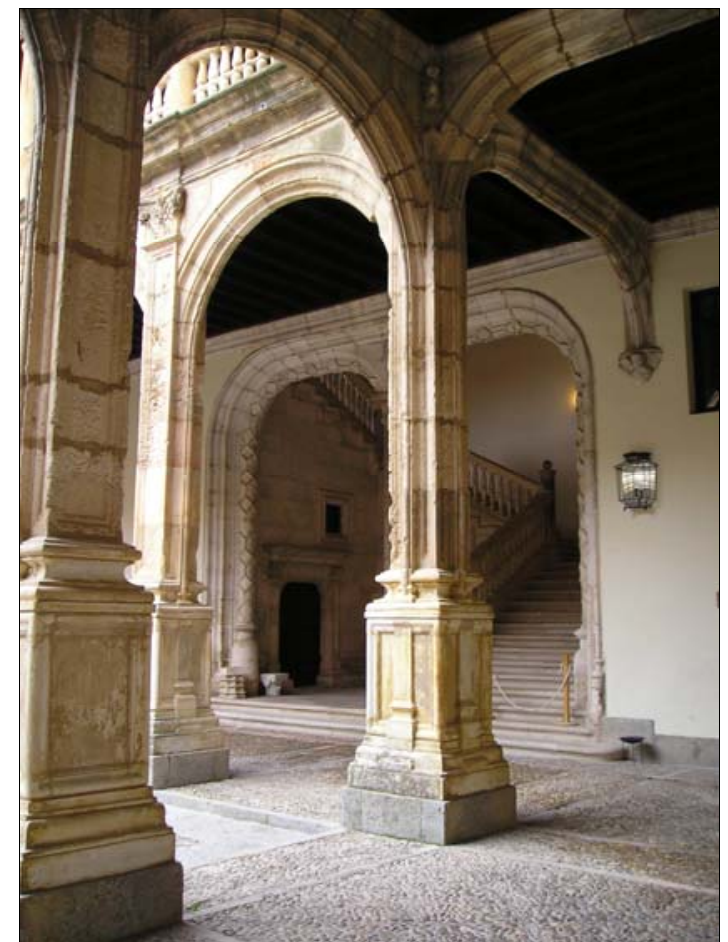

- Fig. 3. Palacio de los condes de Miranda. Vista de la escalera desde el zaguán. Fotog. del autor.

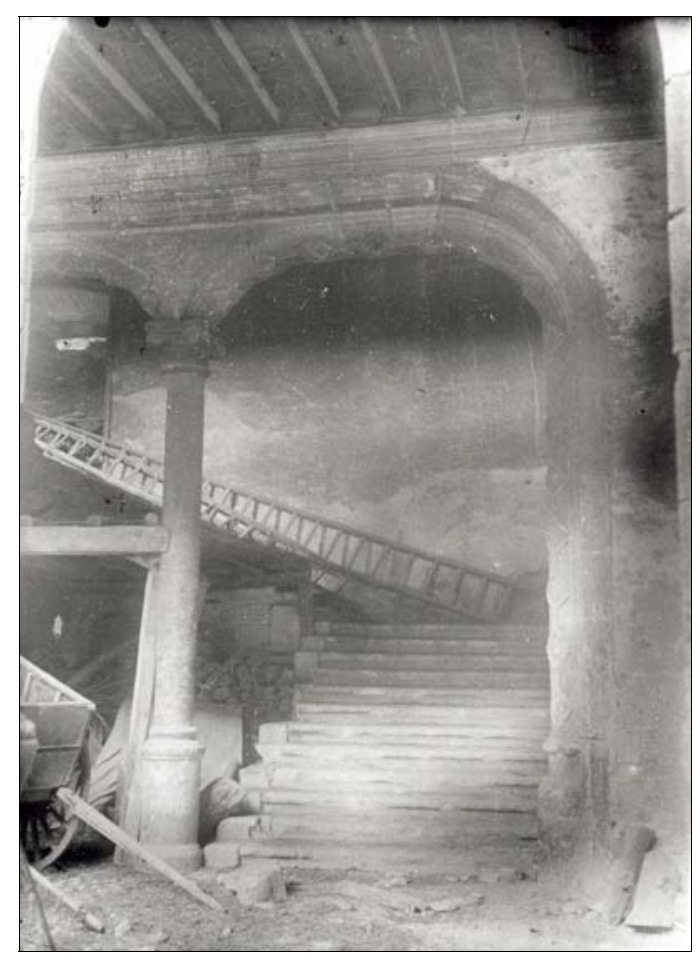

- Fig. 4. Palacio de los condes de Miranda. Vista de la escalera a principios de siglo. ADB. № 4.562 . 


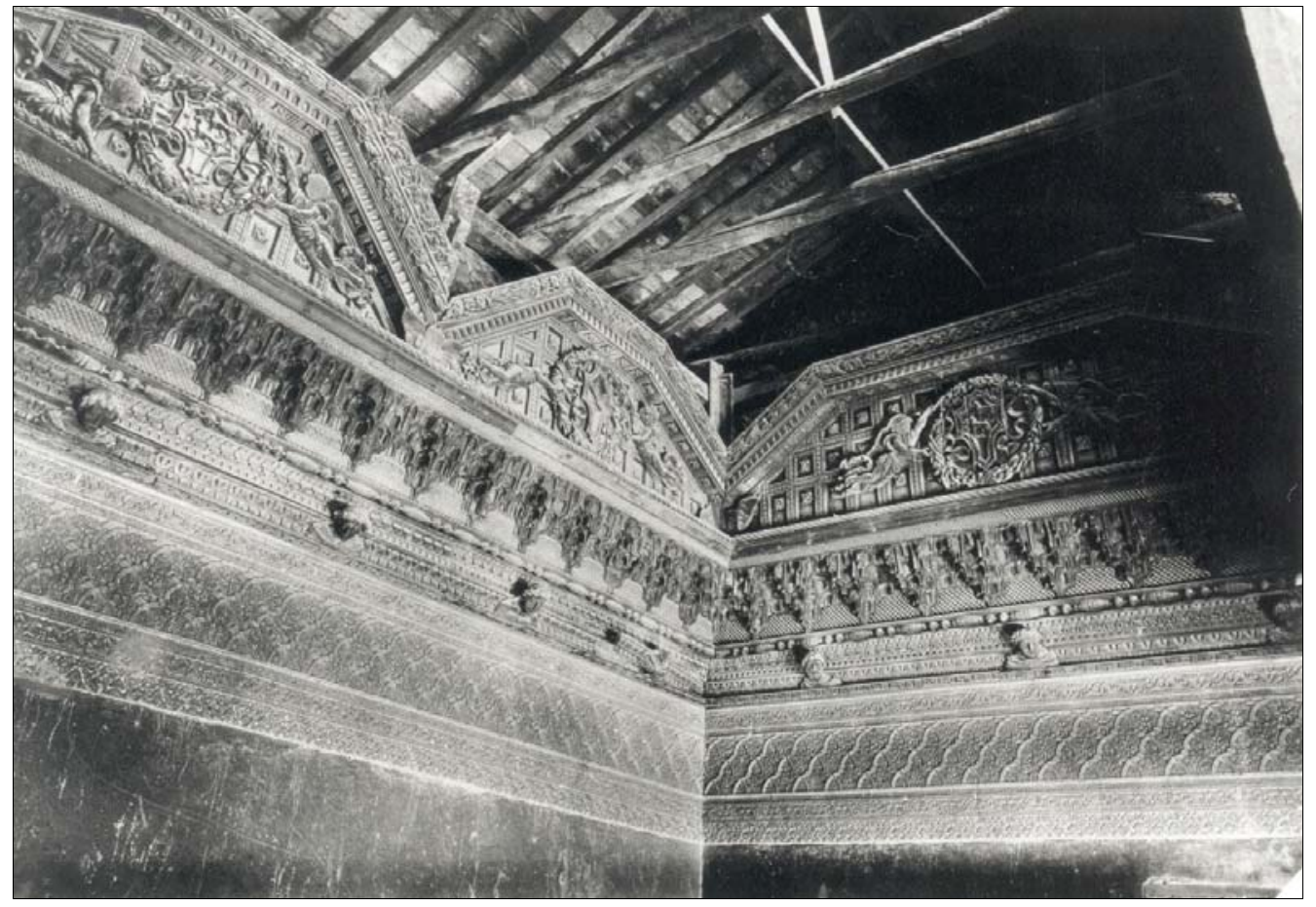

- Fig. 5. Palacio de los condes de Miranda. Artesonado de la escalera a principios de siglo. AMB. FO-2.886.

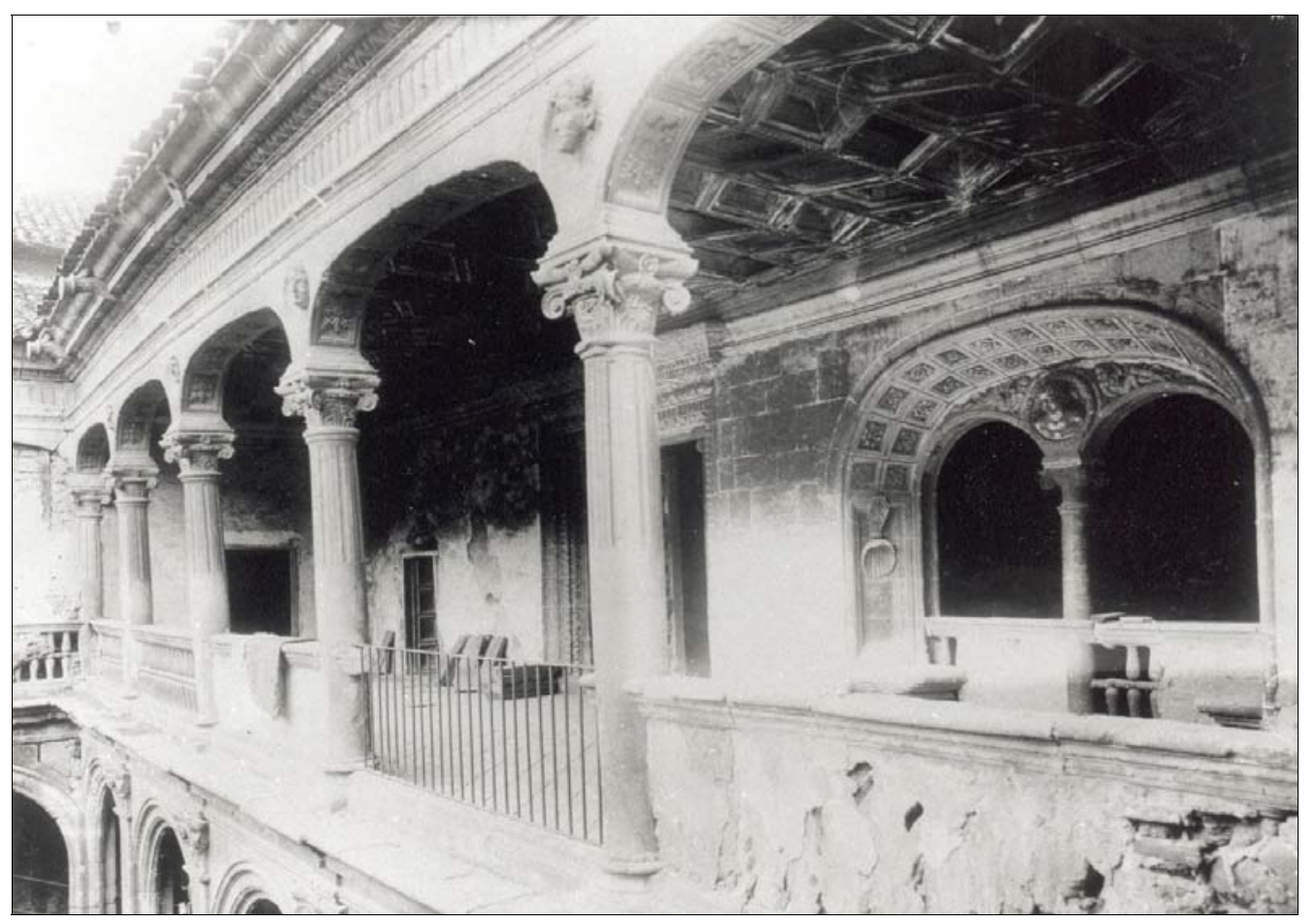

- Fig. 6. Palacio de los condes de Miranda. Vista de la galería alta del patio, tribuna y desembocadura de la escalera. AMB. FO-2.881. 


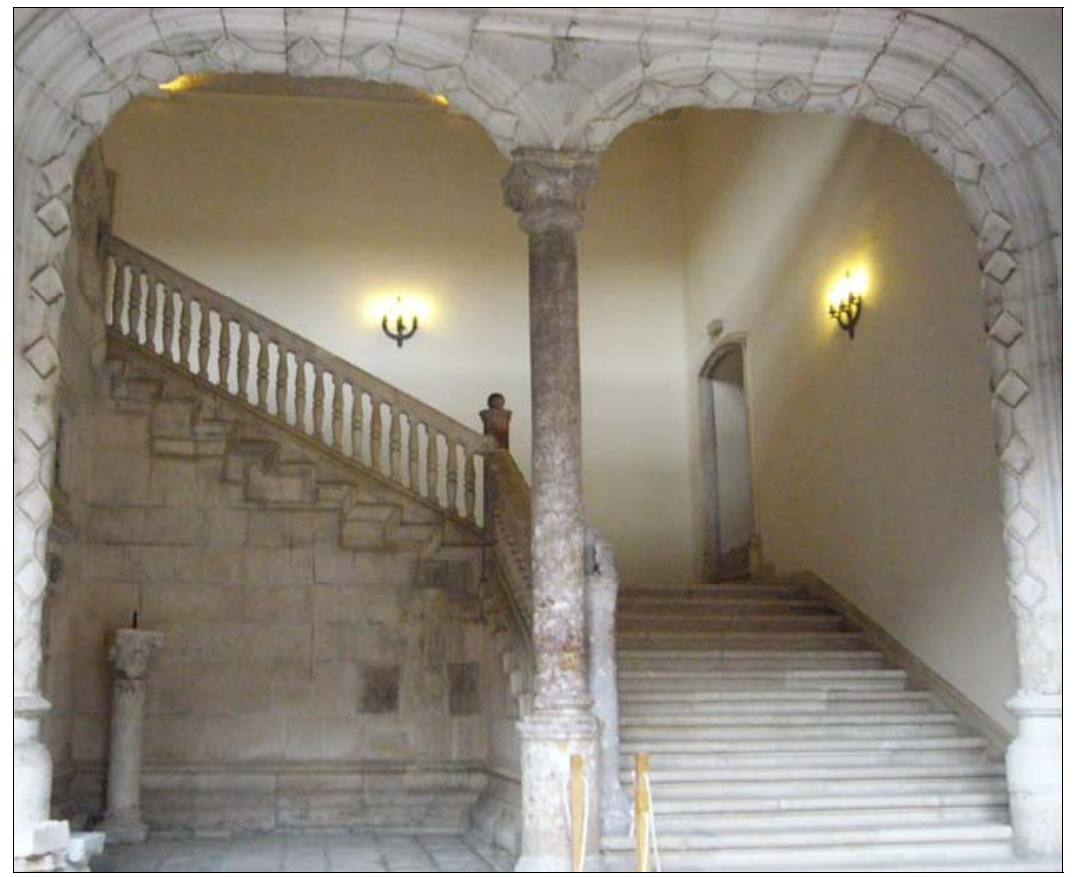

- Fig. 7. Palacio de los condes de Miranda. Vista de la escalera desde el patio. Fotografía del autor.

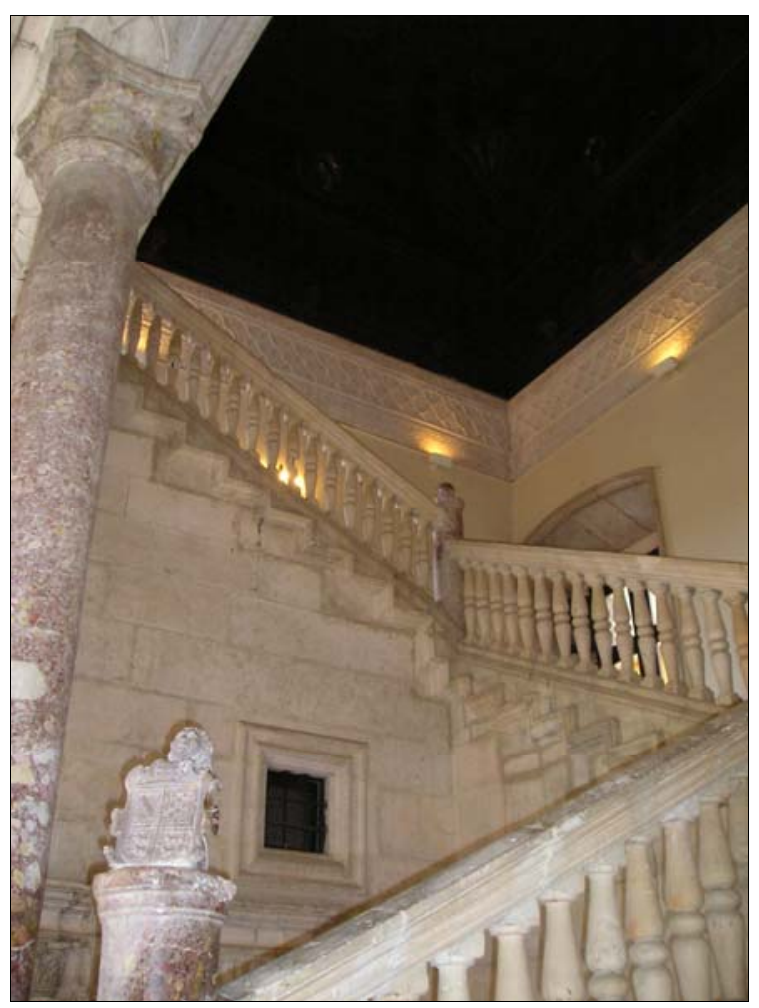

- Fig. 8. Escalera del palacio de los condes de Miranda. Fotografía del autor. 


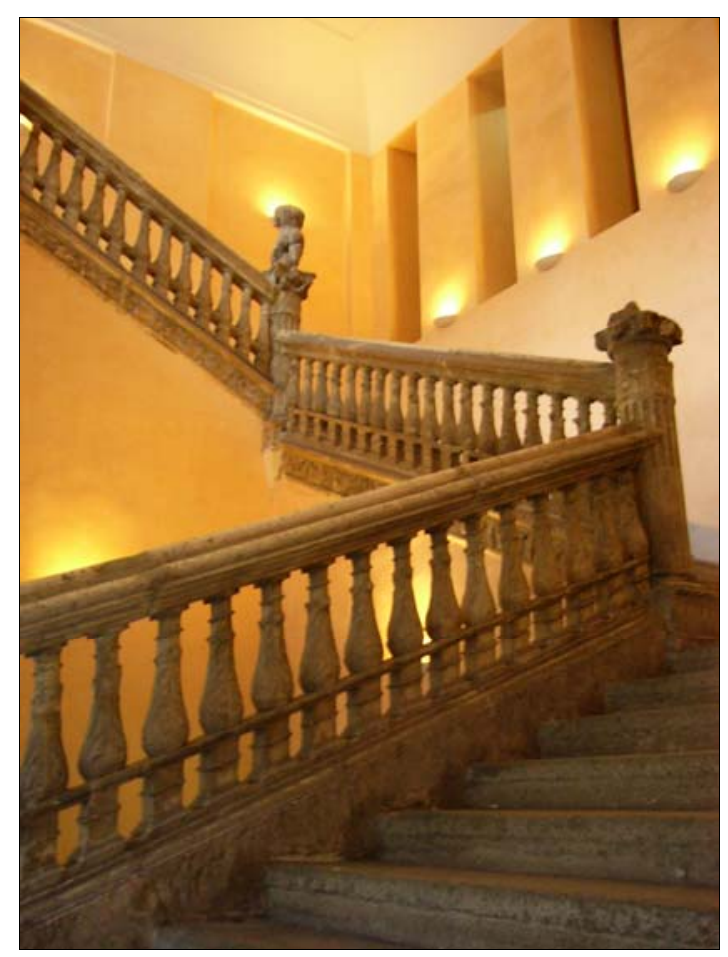

- Fig. 9. Escalera del palacio de los Dueñas, Medina del Campo. Fotografía del autor.

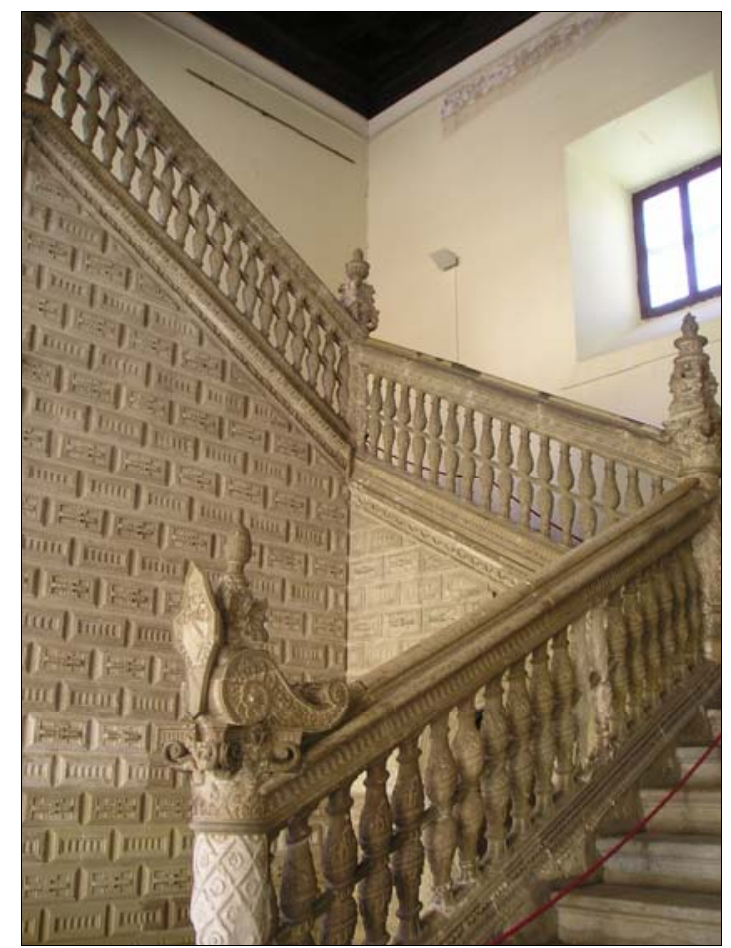

- Fig. 10. Escalera del hospital de la Santa Cruz, Toledo. Fotografía del autor. 


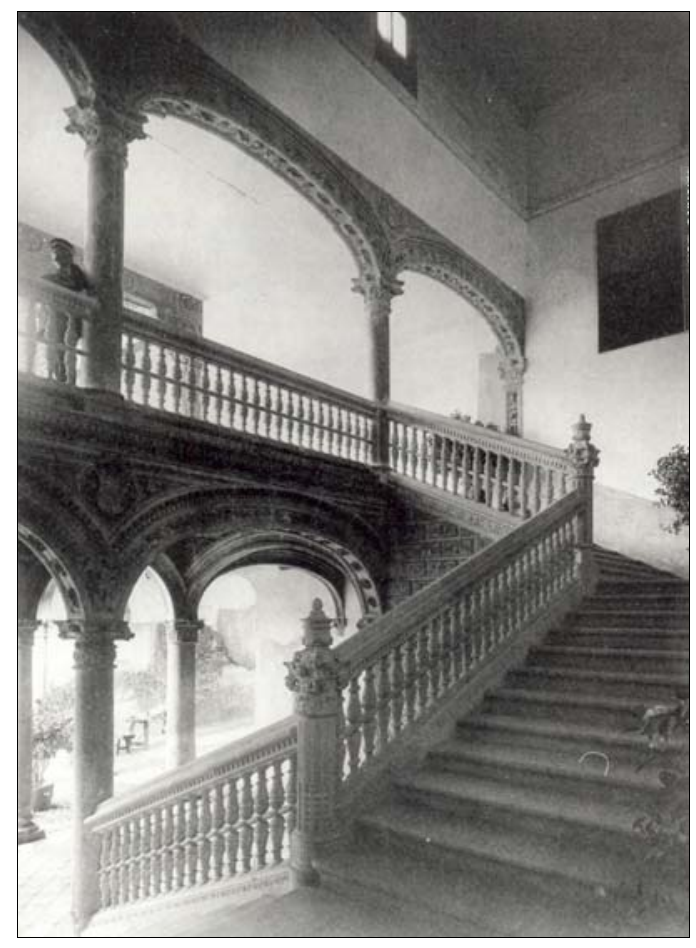

- Fig. 11. Escalera del palacio arzobispal de Alcalá de Henares. M. JUNGHÄNDEL, Die Baukunst Spaniens, II, Dresden, 1893, lám. 105. AMB. $\mathrm{n}^{\mathrm{o}} 13.844$.

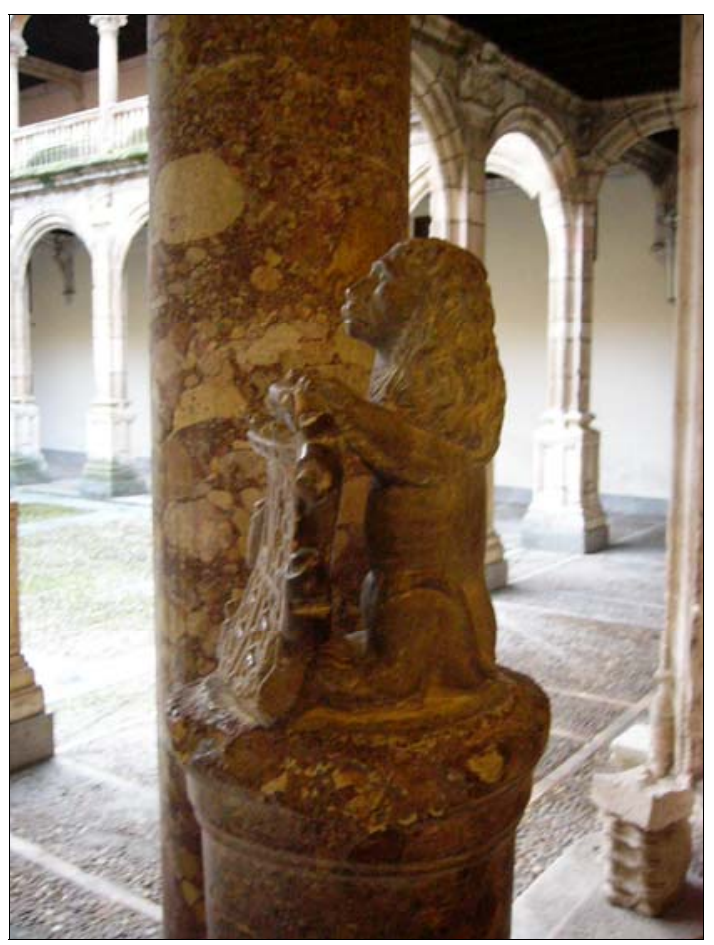

- $\quad$ Fig. 12. Detalle de la escalera del palacio de los condes de Miranda. Fotografía del autor. 